\title{
Fast Reactors and Advanced Light Water Reactors for Sustainable Development
}

\author{
Toshikazu Takeda, ${ }^{1}$ Massimo Salvatores, ${ }^{2}$ Giuseppe Palmiotti, ${ }^{3}$ \\ Kazumi Aoto, ${ }^{4}$ and Katsuhisa Yamaguchi ${ }^{1}$ \\ ${ }^{1}$ Research Institute of Nuclear Engineering, University of Fukui, Tsuruga, Fukui 914-0055, Japan \\ ${ }^{2}$ Commissariat à l'Energie Atomique de Cadarache, 13108 St. Paul-lés-Durance, France \\ ${ }^{3}$ Idaho National Laboratory, Idaho Falls, ID 83415, USA \\ ${ }^{4}$ Japan Atomic Energy Agency, O-arai, Ibaraki 311-1393, Japan \\ Correspondence should be addressed to Toshikazu Takeda,t_takeda@u-fukui.ac.jp
}

Received 23 August 2012; Accepted 23 August 2012

Copyright ( $) 2012$ Toshikazu Takeda et al. This is an open access article distributed under the Creative Commons Attribution License, which permits unrestricted use, distribution, and reproduction in any medium, provided the original work is properly cited.

The importance of nuclear energy, as a realistic option to solve the issues of the depletion of energy resources and the global environment, has been re-acknowledged worldwide. In response to this international movement, the papers compiling the most recent findings in the fields of fast reactors (FR) and advanced light water reactors (LWR) were gathered and published in this special issue.

This special issue compiles six articles, most of which are very meticulously performed studies of the multiyear development of design and assessment methods for large sodium-cooled FRs (SFRs), and two are related to the fuel cycle options that are leading to a greater understanding on the efficient utilization of energy resources.

The Japanese sodium-cooled fast reactor (JSFR) is addressed in two manuscripts. H. Yamano et al. reviewed the current design which adopts a number of innovative technologies in order to achieve economic competitiveness, enhanced reliability, and safety. Their safety assessments of both design basis accidents and severe accidents indicate that the devised JSFR satisfies well their risk target. T. Takeda et al. discussed the improvement of the modeling accuracy for the detailed calculation of JSFR's features in three areas: neutronics, fuel materials, and thermal hydraulics. The verification studies which partly use the measured data from the prototype FBR Monju are also described. Two of these manuscripts deal with those aspects of advanced design of SFR that have hitherto not been explored in great depth.

The paper by G. Palmiotti et al. explored the possibility of using the sensitivity methodologies in the reactor physics field. A review of the methods used is provided, and several examples illustrate the success of the methodology in reactor physics. A new application as the improvement of nuclear basic parameters using integral experiments is also described.

F. Baque et al. reviewed the evolution of the in-service inspection and repair (ISI\&R) capabilities for the SFR under development in France. Associated needs are being defined through an iterative method between designers and inspection specialists: adaptation of the SFR design to ISI\&R requirements, validation of the ultrasonic transducers and associated ultrasonic nondestructive examination techniques, and validation of laser repair processes and associated robotic equipment.

F. Gao et al. and W. Ko et al. discussed how to treat the fuel cycle options for the efficient utilization of energy resources. Which type of reactor to be employed, and whether or not to adopt a reprocessing technique for spent fuel, are two key issues to be addressed. SFR and fuel recycling coupled to pyroprocessing gained considerable attention and shows promising advantages. 
By compiling these papers, we hope to broaden the knowledge of our readers with respect to the presented subjects which are all of high importance for sustainable development.

Toshikazu Takeda Massimo Salvatores

Giuseppe Palmiotti Kazumi Aoto Katsuhisa Yamaguchi 

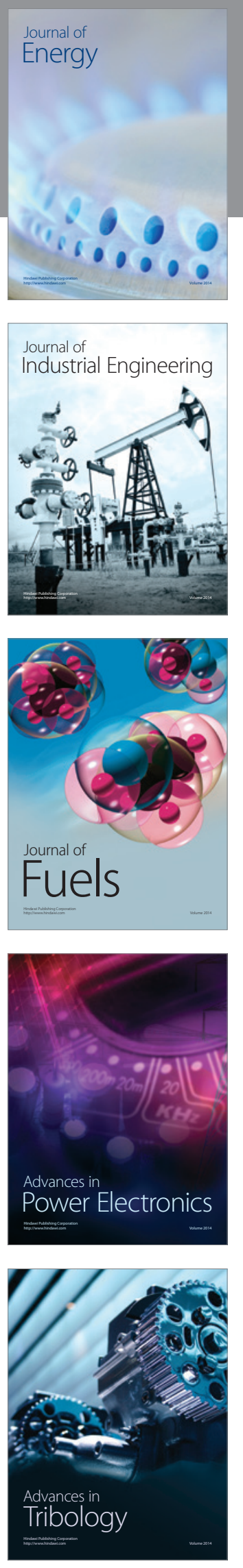
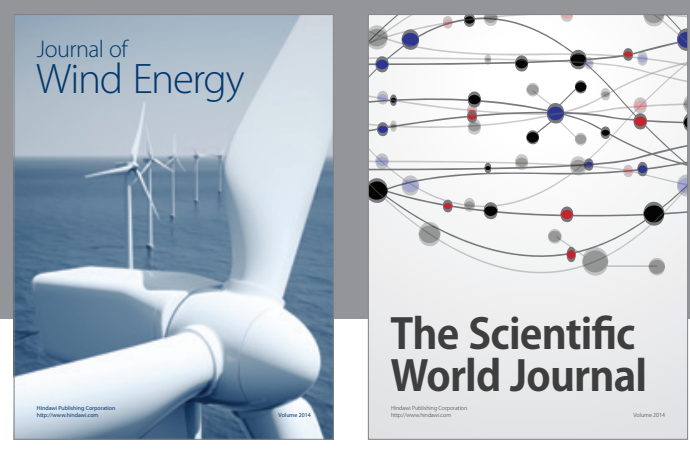

The Scientific World Journal

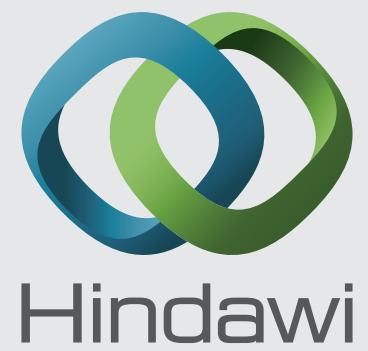

Submit your manuscripts at http://www.hindawi.com
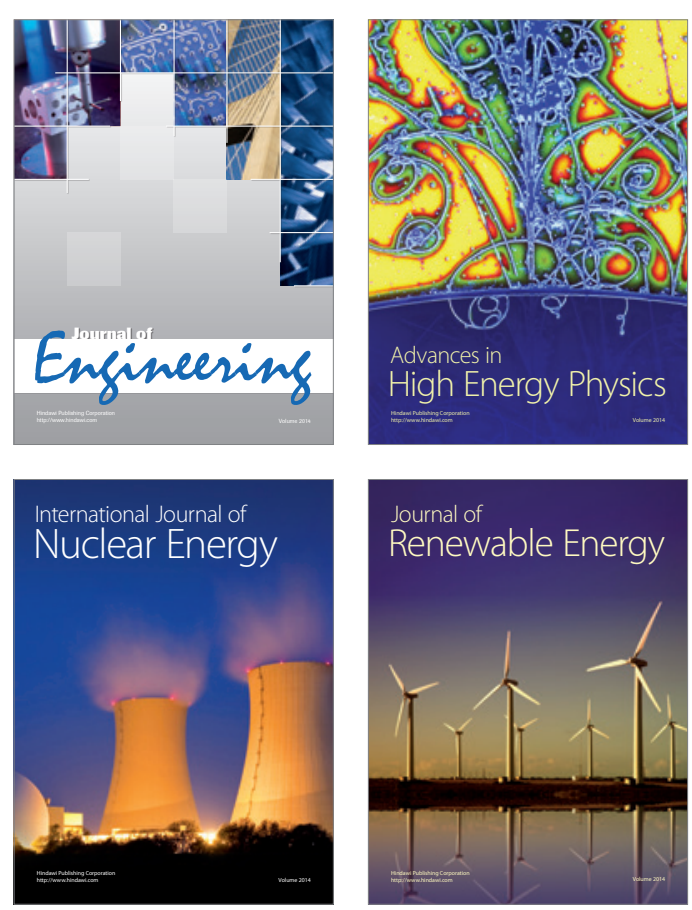

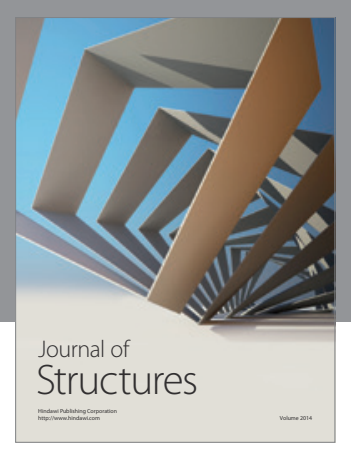

Rotating
Mechinery
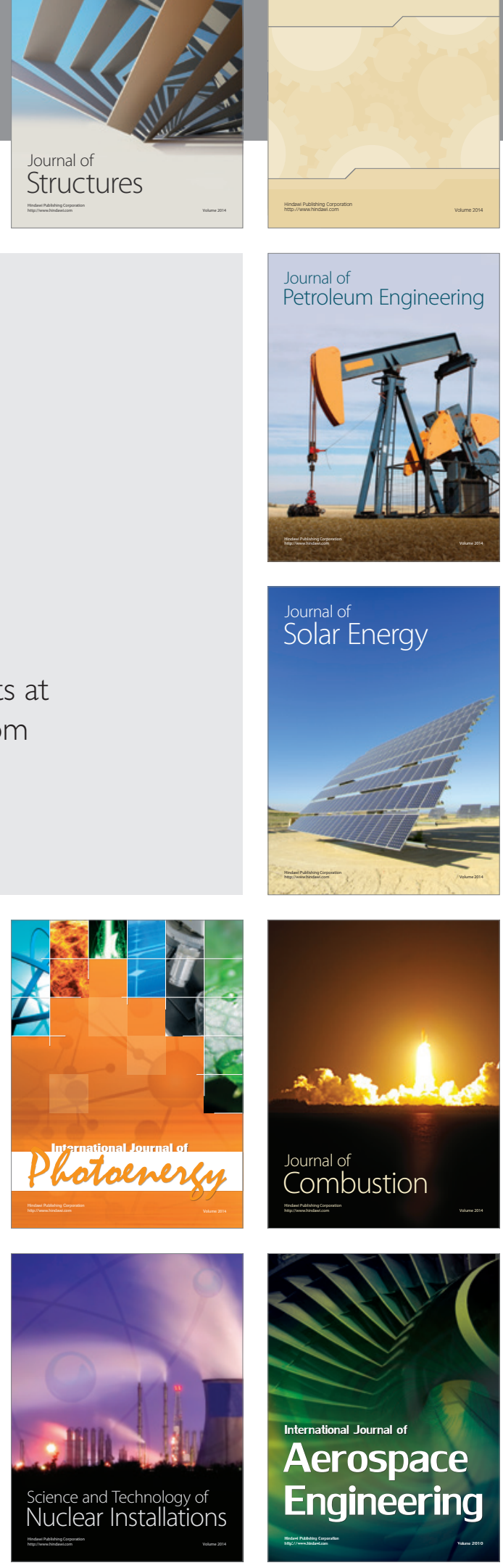